resonantly absorbing multiple photons from the dipole-trap laser (Fig. 1c).

The molecular ions were then captured in the ion trap, where their motion was sympathetically cooled by simultaneously trapped laser-cooled barium ions (Fig. 1d). To avoid secondary reactive or inelastic collisions of the $\mathrm{Rb}_{2}{ }^{+}$ions with the ultracold atoms, the centres of the two traps were separated from one another. The cold molecular ions were localized near the centre of the ion trap, where they could be individually detected by analysing the laser-cooling fluorescence images of the $\mathrm{Ba}^{+}$ ions. An alternative method was also used to detect the formation of molecular ions, relying on monitoring an increased loss of atoms from the optical dipole trap induced by energetic ion-neutral collisions.

By measuring the $\mathrm{Rb}_{2}{ }^{+}$ion production rate as a function of the frequency of the dipole-trap laser, Härter et al. were able to record a resonance-enhanced multiphoton ionization (REMPI) spectrum of the $\mathrm{Rb}_{2}$ molecules. This technique allowed them to map out the molecular quantum states produced in the three-body recombination collisions. In contrast to previous studies, which only probed the most weakly bound molecular states, the signatures of three deeply bound vibrational levels could be identified within the spectral range covered.

The REMPI spectrum also revealed that the molecules were formed with significant rotational excitation - echoing the angular momentum partitioning in the three-body collisions. This result is remarkable given that collisions in the ultracold gas can only occur with vanishing angular momentum, as they would otherwise be suppressed by centrifugal energy barriers.

The study by Härter et al. demonstrates a powerful method for investigating the details of three-body recombination processes. However, the narrow scanning range of the dipole-trap laser used in their experiments limits the investigation to a small subset of all populated molecular states. To enable a stringent comparison with theoretical models, a more comprehensive overview of the level populations needs to be acquired. This could be achieved by ionizing the molecules with an independent, widely tunable laser source. Although the technique using single ions for measuring the molecule production rates is very sensitive, it also requires averaging over a very large number of single-ion events to accumulate sufficient measurement statistics. This approach thus sets a practical constraint on the range of states that can be studied. Using larger ensembles of laser-cooled ions to sympathetically cool a larger number of $\mathrm{Rb}_{2}{ }^{+}$ions in a single experimental run could remove this bottleneck and considerably enhance the scope of the experiments.

Stefan Willitsch is in the Department of Chemistry, University of Basel, Basel 4056, Switzerland. e-mail:stefan.willitsch@unibas.ch

References

1. Burt, E. A. et al. Phys. Rev. Lett. 79, 337 (1997)

2. Esry, B. D., Greene, C. H. \& Burke, J. P. Phys. Rev. Lett. 83, 1751 (1999)

3. Weber, T. et al. Phys. Rev. Lett. 91, 123201 (2003).

4. Chin, C. et al. Rev. Mod. Phys. 82, 1225 (2010).

5. Kraemer, T. et al. Nature 440, 315 (2006)

6. Ferlaino, F. et al. Few-Body Syst. 51, 113 (2011).

7. Härter, A. et al. Nature Phys. 9, 512-517 (2013).

8. Schmid, S., Härter, A. \& Hecker Denschlag, J.

Phys. Rev. Lett. 105, 133202 (2010).

9. Schmid, S. et al. Rev. Sci. Instrum. 83, 053108 (2012).

Published online: 23 June 2013

NANOMECHANICAL RESONATORS

\title{
Spinning oscillators
}

Coupled nanomechanical oscillators can show similar dynamics to two-level systems, and may eventually be used as quantum bits.

\section{Klemens Hammerer}

$\mathrm{T}$ he first computing machines were completely mechanical. Two experiments reported in Nature Physics suggest that future quantum computers might contain some moving mechanical parts too ${ }^{1,2}$. Hajime Okamoto and Thomas Faust and their respective co-workers realize a system of two nanomechanical oscillators exhibiting a significant mutual coupling that is tunable by external fields. The coupling is strong enough to shift and split the resonance frequencies of the two mechanical oscillators by more than their respective linewidths. This enables, with the application of suitable drive fields, a remarkable level of time-domain control over the amplitudes and the relative phase of oscillations. The characteristic timescale of the control pulses exceeds the ring-down time of the oscillators, and therefore permits coherent control of the system. In both experiments the oscillators are excited to large amplitudes to overcome the noisy background created by thermal excitations. As a consequence, the observed dynamics can be fully understood on a classical physics level. However, the techniques demonstrated in the experiments by Faust et al. ${ }^{2}$ and Okamoto et al. ${ }^{1}$ provide important tools for manipulating the quantum dynamics of mechanical two-level systems, if some extra requirements are met.

Recent improvements in nanofabrication have opened the door to nanostructures that exhibit mechanical resonances of exceedingly high quality factors. Voltages applied to electrodes integrated into the nanostructure can tune and actuate the mechanical resonators, piezoelectrically in the case of Okamoto et al. and dielectrically in the experiment by Faust et al. The same mechanism also provides sensitive detection of the amplitude and phase of the mechanical oscillation. A crucial step accomplished in the two experiments was engineering two mechanical modes with strong and tunable coupling between them. Okamoto and colleagues achieved this using a fixed structural coupling between two doubly clamped nanobeams subject to a suitable parametric drive. Faust et al. realized a tunable coupling of two modes of a single nanobeam by means of the cross derivatives of a strong inhomogeneous electric field generated by the electrodes. In both cases the strong-coupling regime was reached - where the coupling strength exceeds the intrinsic linewidth of the mechanical resonances - by a factor of four in Okamoto's and a remarkable four orders of magnitude in Faust's experiment. Strong coupling was evident from a normal-mode splitting of the two resonances as measured in the readout voltages when appropriate pump voltages 
a

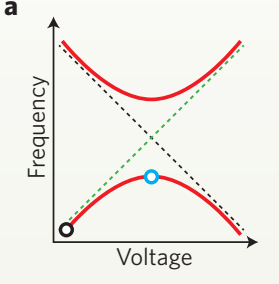

b
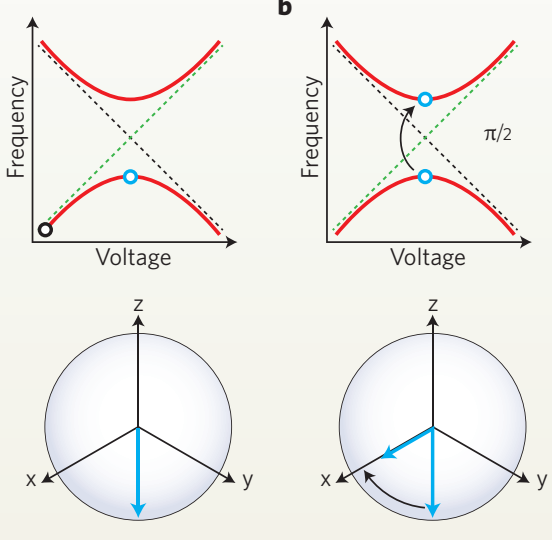

c
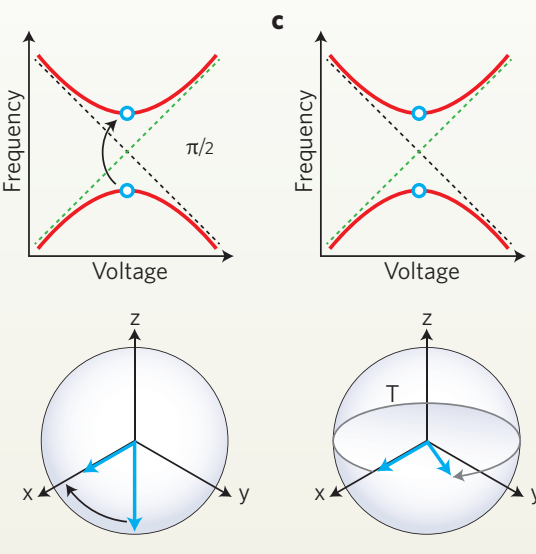

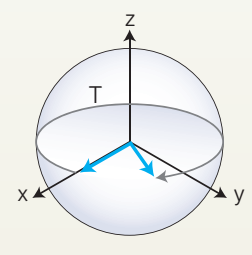

d
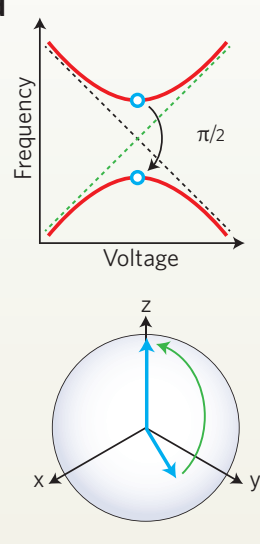

Figure 1 | Coupled nanomechanical oscillators and the dynamics of spin. A Ramsey sequence, similar to that common in NMR, can be applied to the nanomechanical oscillators with suitable control pulses. a, At low control voltage the two mechanical oscillators are effectively decoupled. One of them is driven to a large amplitude (top, black dot) and the voltage is then ramped up to the point of avoided crossing (blue dot) of the normal modes (red lines). Dashed lines indicate the frequencies of uncoupled oscillators. The coupled-oscillator system is thereby initialized in the 'spin-down' state (blue arrow) in the equivalent spin-dynamics diagram (bottom). $\mathbf{b}, A \pi / 2$ pulse rotates the spin into the equatorial plane; that is, half of the initial amplitude of the lower normal mode is transferred to the upper one (blue dots). $\mathbf{c}_{\boldsymbol{r}}$ The spin freely rotates about the $z$ axis for a time $T$ - the relative phase of oscillations of the two normal modes shifts with respect to the local oscillator supplying the $\pi / 2$ pulses. $\mathbf{d}$, A final $\pi / 2$ pulse rotates the spin back to the $z$ axis (blue dots). The length of the spin vector shrinks as the initial amplitude damps out. Normal-mode splitting and Rabi frequency of control pulses therefore need to be larger than the oscillator's damping rate, as is accomplished in the experiments of Okamoto et al. and Faust etal.

actuated the coupled system. This coupling provided the basis for another compelling achievement: both groups demonstrated fast and versatile time control of the two coupled mechanical modes by application of a suitable electric-pulse sequence (Fig. 1). An amplitude excitation imposed initially on one of the modes moved coherently back and forth between the two modes. Selecting the right frequency, intensity and duration of drive pulses enabled the researchers to fully control the relative weight of the amplitude and the relative phase of the oscillations.

These protocols are a perfect analogy for the coherent control of (ensembles of) spins, as achieved in NMR with magnetic pulses, for example. The Bloch vector describes the state of a spin (ensemble) by its spherical coordinates on the Bloch sphere. In the experiments of Faust ${ }^{2}$ and Okamtoto ${ }^{1}$, the two angles of the equivalent Bloch vector encode only the relative amplitude and phase of the mechanical oscillators. The authors applied arbitrary rotations on the Bloch sphere using their control of these two degrees of freedom. This allowed them to perform the standard pulse sequences familiar in coherent spin control such as Rabi oscillations, Ramsey sequences or Hahn echoes. For example, Okamoto et al. observe up to eight Rabi flops before the oscillations ring down and the length of the equivalent Bloch vector vanishes. The Rabi frequencies achieved by Faust et al. exceeded the amplitude decay rate by two orders of magnitude.

Whereas the picture of the Bloch vector gives an accurate understanding of the results, it is important to note that the observed physics is entirely classical, as the authors emphasize. All that matters in the experiments is the coherent amplitude of the two coupled oscillators, and at no point are quantum superposition states generated. However, the clear demonstration of strong coupling between the oscillators and the ensuing level of coherent control over their dynamics is still very exciting from a quantum physics perspective. The requirements for turning this system into a real quantum mechanical two-level system, that is a quantum bit or 'qubit', are challenging but clear. First, the temperature must be decreased further (or the mechanical quality factor increased) such that the quantum coherence time comes close to the oscillator's ringdown time. Second, the system needs to be initialized in a state in which a single phonon is shared between the two oscillators, similar to a recent study ${ }^{3}$. The equivalent optical experiment encoding a qubit in the state of a single photon shared between two modes of the electromagnetic field - is now routine in numerous photonics laboratories. The techniques developed by Okamoto, Faust and their co-workers open a new vista to a truly quantum mechanical phononic - realization of quantum bits, for which single-qubit control is now demonstrated. Together with recent theoretical work along this line $e^{4,5}$, these results add momentum to nanomechanical implementations of quantum information processors.

Klemens Hammerer is at the Institute for Theoretical Physics and the Institute for Gravitational Physics (Albert Einstein Institut), Leibniz Universität Hannover, Callinstraße 38, D-30167 Hannover, Germany.

e-mail: Klemens.Hammerer@itp.uni-hannover.de

\section{References}

1. Okamoto, H. et al. Nature Phys. 9, 480-484 (2013).

2. Faust, T., Rieger, J., Seitner, M., Kotthaus, J. \& Weig, E. Nature Phys. 9, 485-488 (2013).

3. O'Connell, A. D. et al. Nature 464, 697-703 (2010).

4. Rips, S. \& Hartmann, M. J. Phys. Rev. Lett. 110, 120503 (2013).

5. Stannigel, K. et al. Phys. Rev. Lett. 109, 013603 (2012).

Published online: 2 July 2013 